\title{
Generalized Linear Mixing Rule for Classical Coulomb Mixtures
}

\author{
A. I. Chugunov* \\ Ioffe Institute, Politekhnicheskaya 26, 194021 Saint-Petersburg, Russia \\ Received 14 October 2011, revised 06 December 2011, accepted 13 December 2011 \\ Published online 27 February 2012
}

Key words Equation of state; strongly coupled plasmas.

It is shown that the Coulomb energy $U$ of fully ionized ionic mixture can be written as a sum over partial contributions of ion species $j: U=T \sum_{j} N_{j} u\left(\Gamma_{j}, y_{j}\right)$ (generalized linear mixing rule). In contrast to the traditional linear mixing rule $U_{\mathrm{LM}}=T \sum_{j} N_{j} u_{\mathrm{OCP}}\left(\Gamma_{j}\right)$, applicable for strong coupling, the partial contribution function $u$ depends not only on $\Gamma_{j}$, but on an additional parameter $y_{j}=\left(r_{\mathrm{D}} / r_{\mathrm{D}}^{\mathrm{OCP}}\right)^{2}$ also. Here $r_{\mathrm{D}}$ and $r_{\mathrm{D}}^{\mathrm{OCP}}$ are Debye radiuses in the mixture and in the one component plasma at coupling parameter $\Gamma_{j}$, correspondingly. The parameter $y_{j}$ does not depend on a specific composition of the mixture, but on the Debye radius $r_{\mathrm{D}}$ only, making function $u\left(\Gamma_{j}, y_{j}\right)$ universal. The generalized linear mixing rule can be applied at any coupling parameter, if ionic mixture is not crystallized. It reproduces results of the Debye-Hückel theory at weak coupling and traditional linear mixing rule at strong coupling. It can be easily applied to the complicated mixtures, composed of a large number of ion species. Since $y_{j}$ is temperature independent, the Coulomb contribution to Helmholtz free energy of the mixture can also be presented in a form of generalized linear mixing rule.

(c) 2012 WILEY-VCH Verlag GmbH \& Co. KGaA, Weinheim

\section{Introduction and main equations}

In this paper we discuss strongly coupled classical Coulomb mixtures composed of a set of ion species with charges $Z_{j}$ e immersed into neutralizing nonpolarisable background of electrons. Let $n_{j}$ and $N_{j}$ to be number density and total number of ions of type $j$, correspondingly. The electroneutrality fix electron number density $n_{\mathrm{e}}=\sum_{j} Z_{j} n_{j}$. Equation of state (EOS) of such a system at gaseous and liquid phases was recently fitted to results of Monte Carlo (MC) and hypernetted-chain (HNC) simulations with high accuracy [1] (hereafter PCCDWR EOS). But this fit is somewhat artificial and more physical approximations may be useful. For this aim we suggest representation of EOS in form of generalized linear mixing (GLM) rule for Coulomb energy of the system:

$$
U=T \sum_{j} N_{j} u\left(\Gamma_{j}, y_{j}\right)
$$

Here $u\left(\Gamma_{j}, y_{j}\right)$ is partial contribution function, which is universal for all Coulomb mixtures and depend just on two parameters: effective coupling parameter of correspondent component $\Gamma_{j}=Z_{j}^{2} e^{2} /\left(a_{j} T\right)$ and composition dependent parameter

$$
y_{j}=\left(\frac{r_{\mathrm{D}}}{r_{\mathrm{D}}^{\mathrm{OCP}}}\right)^{2}=\frac{3 \Gamma_{j} r_{\mathrm{D}}^{2}}{a_{j}^{2}}=\frac{Z_{j}\langle Z\rangle}{\left\langle Z^{2}\right\rangle} .
$$

Here Debye radius $r_{\mathrm{D}}=\left[4 \pi \sum n_{j} Z_{j}^{2} e_{j}^{2} / T\right]^{-1 / 2}$ and $r_{\mathrm{D}}^{\mathrm{OCP}}=a_{j} / \sqrt{3 \Gamma_{j}}$ is Debye radius in one component plasma (OCP) at coupling parameter $\Gamma_{j}$. In addition, $a_{j}=Z_{j}^{1 / 3} a_{\mathrm{e}}=Z_{j}^{1 / 3}\left(4 \pi n_{\mathrm{e}} / 3\right)^{-1 / 3}$. For future discussion let us introduce number fractions of each ion type $x_{j}=N_{j} / N$, total number of ions $N=\sum_{j} N_{j}$ and the mean coupling parameter of the mixture $\Gamma=\sum_{j} x_{j} \Gamma_{j}=\left\langle Z^{5 / 3}\right\rangle e^{2} /\left(a_{\mathrm{e}} T\right)$.

The idea of GLM representation comes from well known (traditional) linear mixing (LM) rule (see [2-5], for example), which suggest $U$ to be equal to $U_{\mathrm{LM}}=T \sum_{j} N_{j} u_{\mathrm{OCP}}\left(\Gamma_{j}\right)$.

* Corresponding author. E-mail: andr.astro@mail.ioffe.ru, Phone: +78122927180 , Fax: +7 8122971017 
It is applicable for strong coupling $\Gamma \gg 1$. Here, the energy $u_{\mathrm{OCP}}\left(\Gamma_{j}\right)$ is temperature normalized OCP Coulomb energy per one ion at coupling parameter $\Gamma_{j}$. This function is well discussed and we will apply approximation suggested at [6] with parameter set fitted to MC results of paper [7]. At GLM representation the partial contribution function $u$ depends not only on $\Gamma_{j}$, but on an additional parameter $y_{j}$ providing thus possibility to describe mixtures at weak coupling. Really, at $\Gamma \ll 1$ the Coulomb energy of the mixture can be calculated within Debye-Hückel theory. It gives

$$
U_{\mathrm{DH}}=-\sum_{j} N_{j} \frac{Z_{j}^{2} e^{2}}{r_{\mathrm{D}}}=-\sum_{j} N_{j}\left(\frac{3 \Gamma_{j}^{3}}{4 y_{j}}\right)^{1 / 2} .
$$

This formula agree with GLM, but can not be written in form of LM rule.

Two previous attempts to generalize linear mixing rule should be mentioned. Rosenfeld [3] suggest representation of the EOS of Coulomb mixture which correspond to GLM partial contribution function $u\left(\Gamma_{j}, y_{j}\right)=$ $y_{j} u_{\mathrm{OCP}}\left(\Gamma_{j} / y_{j}\right)$. It well agree with DH limit, but, unfortunately not enough accurate at strong coupling. At [4] he generalize linear mixing rule to a case of strongly coupled Yukawa systems. Here we concentrate on pure Coulomb systems only, and treat $u\left(\Gamma_{j}, y_{j}\right)$ as a free function with two restrictions: (a) to agree with LM at $\Gamma \gg 1$, partial contribution functions $u\left(\Gamma_{j} \gg 1, y_{j}\right) \approx u_{\mathrm{OCP}}\left(\Gamma_{j}\right)$ being almost independent on $y_{j}$; (b) to agree with DH theory at weak coupling $\Gamma \ll 1$, one should have $u\left(\Gamma_{j} \rightarrow 0, y_{j}\right) \approx-\left[3 \Gamma_{j}^{3} /\left(4 y_{j}\right)\right]^{1 / 2}$. Our aim is to check applicability of GLM representation of EOS at intermediate coupling by fitting of $u\left(\Gamma_{j}, y_{j}\right)$ function. The accuracy achieved in this fitting is discussed in Sec. 3.

Since parameters $y_{j}$ do not depend on the temperature, the possibility of GLM representation of Coulomb energy (1) guarantee accuracy of GLM representation for the Coulomb contribution to Helmholtz free energy $F$ :

$$
F=T \sum_{j} N_{j} f\left(\Gamma_{j}, y_{j}\right)
$$

with $f\left(\Gamma_{j}, y_{j}\right)=\int_{0}^{\Gamma_{j}} u\left(\Gamma_{j}^{\prime}, y_{j}\right) / \Gamma_{j}^{\prime} \mathrm{d} \Gamma_{j}^{\prime}$.

\section{Properties of the generalized linear mixing rule}

Let us discuss binary ionic mixtures (BIM) and demonstrate two important properties of GLM representation of EOS: (1) Arbitrary EOS can not be presented in GLM form. So possibility of GLM representation is an important feature of EOS; (2) Just the same GLM EOS can be presented by different functions $u\left(\Gamma_{j}, y_{j}\right)$ [see Eq. (8)]. However, for $y=1$ this function is well defined: $u\left(\Gamma_{j}, 1\right)=u_{\mathrm{OCP}}\left(\Gamma_{j}\right)$.

It is easy to show that composition of binary mixture $\left(x_{1}, x_{2}=1-x_{1}\right.$, and $\left.Z_{2} / Z_{1}\right)$ is unambiguously defined by a couple of parameters $\left(y_{1}, y_{2}\right)$ :

$$
\frac{Z_{2}}{Z_{1}}=\frac{y_{2}}{y_{1}}, \quad x_{1}=\frac{y_{2}\left(y_{2}-1\right)}{\left(y_{2}-y_{1}\right)\left(y_{2}+y_{1}-1\right)} .
$$

The conditions $0<x_{1}<1$ and $0<x_{2}<1$ restricts available parameter space to $0<y_{1}<1$ and $1<y_{2}$. Here we suppose $Z_{1}<Z_{2}$ for definiteness. So, we can characterize BIM by a set of three numbers: $\left(\Gamma_{1}, y_{1}, y_{2}\right)$.

Generalized linear mixing rule for BIM can be written in form:

$$
u\left(\Gamma_{1}, Z_{2} / Z_{1}, x_{1}, x_{2}\right)=x_{1}\left(y_{1}, y_{2}\right) u\left(\Gamma_{1}, y_{1}\right)+\left[1-x_{1}\left(y_{1}, y_{2}\right)\right] u\left(\Gamma_{1}\left(y_{2} / y_{1}\right)^{5 / 3}, y_{2}\right)
$$

Here $u\left(\Gamma_{1}, Z_{2} / Z_{1}, x_{1}, x_{2}\right)=U /\left(T \sum_{j} N_{j}\right)$ and $U$ is the Coulomb energy of this mixture.

Let us fix three parameters $\Gamma_{1}, y_{1}$ and $y_{2}\left(y_{1}<1<y_{2}\right)$ and add to them two arbitrary numbers $y_{3}<1$ and $y_{4}>1$. This set can be applied to define four different binary mixtures, which correspond to the following triples of parameters: $\left(\Gamma_{1}, y_{1}, y_{2}\right),\left(\Gamma_{1}, y_{1}, y_{4}\right),\left(\Gamma_{3}, y_{3}, y_{2}\right),\left(\Gamma_{3}, y_{3}, y_{4}\right)$. Here $\Gamma_{3}=\Gamma_{1}\left(y_{3} / y_{1}\right)^{5 / 3}$. Let us write a generalized linear mixing rule for each of these binary mixtures:

$$
\begin{aligned}
u\left(\Gamma_{1}, y_{2} / y_{1}, x_{1}\left(y_{1}, y_{2}\right), x_{2}\left(y_{1}, y_{2}\right)\right)=x_{1}\left(y_{1}, y_{2}\right) u\left(\Gamma_{1}, y_{1}\right)+x_{2}\left(y_{1}, y_{2}\right) u\left(\Gamma_{2}, y_{2}\right), \\
u\left(\Gamma_{1}, y_{4} / y_{1}, x_{1}\left(y_{1}, y_{4}\right), x_{2}(y 1, y 4)\right)=x_{1}\left(y_{1}, y_{4}\right) u\left(\Gamma_{1}, y_{1}\right)+x_{2}\left(y_{1}, y_{4}\right) u\left(\Gamma_{4}, y_{4}\right), \\
u\left(\Gamma_{3}, y_{2} / y_{3}, x_{1}\left(y_{3}, y_{2}\right), x_{2}\left(y_{3}, y_{2}\right)\right)=x_{1}\left(y_{3}, y_{2}\right) u\left(\Gamma_{3}, y_{3}\right)+x_{2}\left(y_{3}, y_{2}\right) u\left(\Gamma_{2}, y_{2}\right), \\
u\left(\Gamma_{3}, y_{4} / y_{3}, x_{1}\left(y_{3}, y_{4}\right), x_{2}\left(y_{3}, y_{4}\right)\right)=x_{1}\left(y_{3}, y_{4}\right) u\left(\Gamma_{3}, y_{3}\right)+x_{2}\left(y_{3}, y_{4}\right) u\left(\Gamma_{4}, y_{4}\right) .
\end{aligned}
$$


Here $\Gamma_{j}=\Gamma_{1}\left(y_{j} / y_{1}\right)^{5 / 3}, x_{2}\left(y_{1}, y_{2}\right)=1-x_{1}\left(y_{1}, y_{2}\right)$ and $x_{1}\left(y_{1}, y_{2}\right)$ is given by Eq. (5). If, the BIM EOS $f_{\mathrm{BIM}}\left(\Gamma_{1}, Z_{2} / Z_{1}, x_{1}, x_{2}\right)$ is known, Eqs. (7) can be treated as a system of four equations on the values of partial contribution function $u$ at correspondent points $\left[u\left(\Gamma_{1}, y_{1}\right), u\left(\Gamma_{2}, y_{2}\right), u\left(\Gamma_{3}, y_{3}\right), u\left(\Gamma_{4}, y_{4}\right)\right]$. However, as it can be shown analytically, this system is degenerate for any set of values $y_{j}, j=1 . .4$. As a result, Eq. (7) can not be solved in a case of arbitrary equation of state. So, the first statement has been proofed and an arbitrary equation of state can not be presented in form of generalized linear mixing rule.

Let us assume, that we find GLM representation of EOS by partial contribution function $u_{0}\left(\Gamma_{j}, y_{j}\right)$, which is enough accurate for Coulomb mixtures and we accept it. But degeneracy of system (7) suggest what choice of partial contribution function $u\left(\Gamma_{j}, y_{k}\right)$ is not unique - exactly the the same GLM EOS can be presented by different partial contribution functions $u\left(\Gamma_{j}, y_{j}\right)$. Really, let us take an arbitrary number $\tilde{y} \neq 1$ and an arbitrary one parameter function $\delta u(\tilde{\Gamma})$ and perform following renormalization of the partial contribution function:

$$
u\left(\Gamma_{j}, y_{j}\right)=u_{0}\left(\Gamma_{j}, y_{j}\right)+\delta u\left(\Gamma_{j} \frac{\tilde{y}^{5 / 3}}{y_{j}^{5 / 3}}\right) \frac{y_{j}\left(1-y_{j}\right)}{\tilde{y}(1-\tilde{y})} .
$$

As can be easily shown, such function $u\left(\Gamma_{j}, y_{j}\right)$ correspond to exactly the same EOS, as initial $u_{0}\left(\Gamma_{j}, y_{j}\right)$. Note, the OCP correspondent partial function $u(\Gamma, 1)$ is well defined $u(\Gamma, 1)=u_{\mathrm{OCP}}(\Gamma)$ and can not be changed by rescaling relation (8). It is important to stress, that scaling relation (8) conserve EOS not only for BIM, but for multicomponent systems with arbitrary number of constituents.
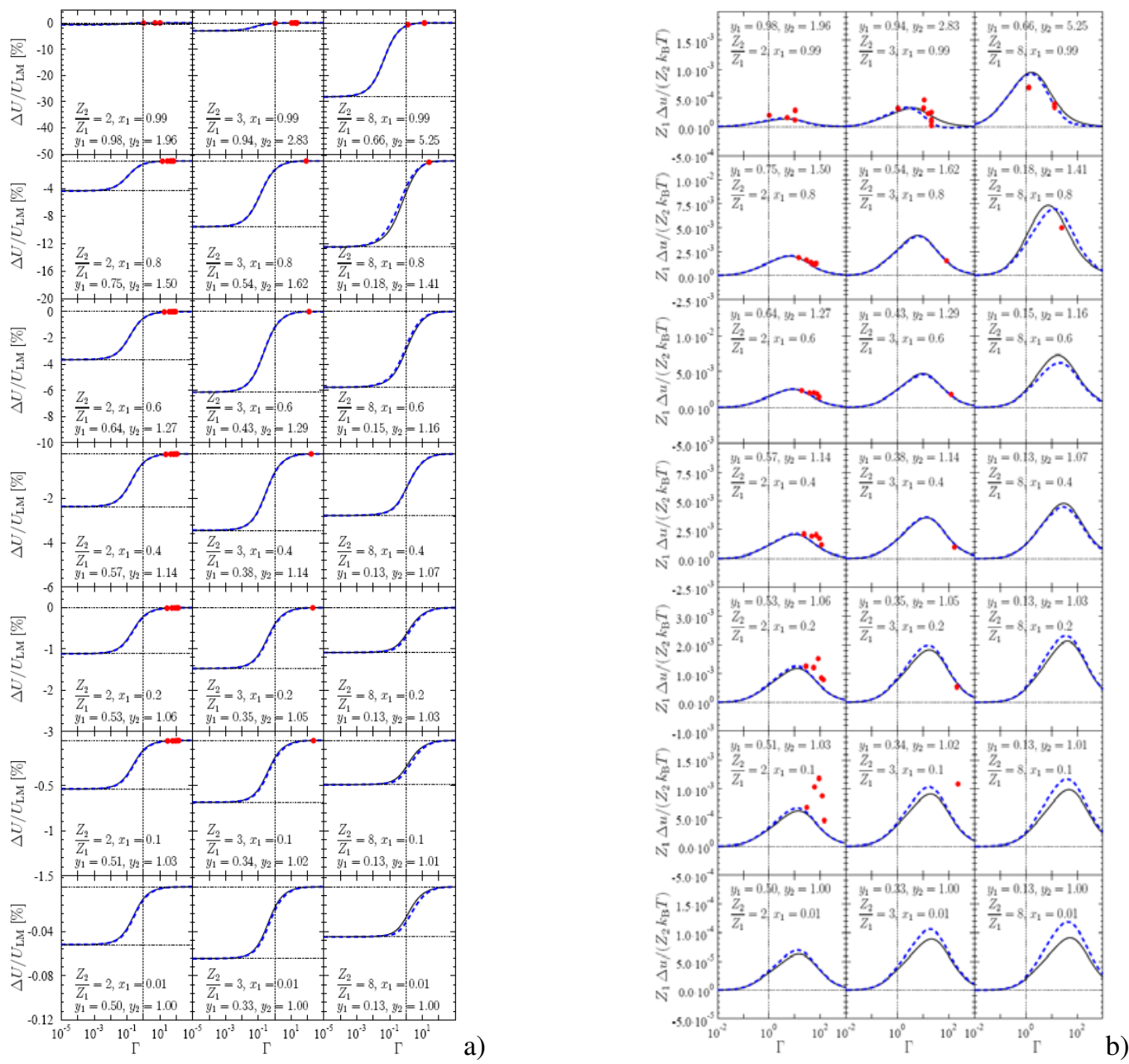

Fig. 1 The relative a) and absolute b) correction to linear mixing rule. See text for details. 


\section{Numerical results}

In this section we present numerical results to illustrate accuracy which can be achieved within GLM representation of EOS.

Figure 1 demonstrates correction to linear mixing rule $\Delta U=U-U_{\mathrm{LM}}$ for a large set of BIMs. Fig.1a represent relative correction $\Delta U / U_{\mathrm{LM}}$. A normalized value of absolute correction $Z_{1} \Delta U /\left(N Z_{2} T\right)$ is shown on panel b. Solid lines correspond to PCCDWR EOS [1] and dashed lines to GLM representation. Dots on both panels are MC data for BIM Coulomb energy from [5]. The mixture parameters are shown on each plot (the $Z_{2} / Z_{1}$ ratio is fixed at each column, while $x_{1}$ is constant in rows).

The agreement between PCCDWR EOS and GLM representation, shown on the figures, seems to be acceptable. Typical difference between them is the same order of magnitude as deviations between MC calculations of Coulomb energy of OCP given at [7] and [8]. We should stress that possibility even better GLM representation of EOS can not be excluded.

\section{Conclusions}

A generalized linear mixing rule (Eq. 1) is suggested to describe EOS of classical Coulomb mixtures in gaseous and liquid phases. The following properties of GLM are shown: (1) Possibility of GLM representation is a feature of the specific class of EOS (see Sec. 2); (2) PCCDWR EOS [1] can be represented in GLM form very accurately (see Fig. 1); (3) The partial contribution function $u\left(\Gamma_{1}, y_{1}\right)$ is not unique for given GLM EOS, but can be rescaled according to Eq. (8); (4) GLM rule can be applied not only for Coulomb energy, but for the Coulomb contribution to Helmholtz free energy also [see Eq. (4)].

The GLM rule was introduced in this paper just on empirical basis, but I hope that it have a simple theoretical motivation which can be important for understanding of the strongly coupled plasma physics.

Acknowledgements I am grateful to D.A. Baiko, H.E. DeWitt, A.Y. Potekhin and W. Ebeling for stimulating discussions. This work was partially supported by the Russian Foundation for Basic Research (grant 11-02-00253-a), by the State Program "Leading Scientific Schools of Russian Federation" (grant NSh 3769.2010.2), by the President grant for young Russian scientists (MK-5857.2010.2) and by the RAS Presidium Programme "Support for Young Scientists".

\section{References}

[1] A. Y. Potekhin et al., Phys. Rev. E 80, 047401 (2009).

[2] J. P. Hansen and P. Villefosse, Phys. Rev. Lett 37, 391 (1976).

[3] Y. Rosenfeld, Phys. Rev. A 26, 3622 (1982).

[4] Y. Rosenfeld, Phys. Rev. E 47, 2676 (1993).

[5] H. DeWitt and W. Slattery, Contrib. Pasma Phys. 43, 279 (2003).

[6] A.Y. Potekhin and G. Chabrier, Phys. Rev. E 62, 8554 (2000).

[7] H.E. DeWitt and W. Slattery, Contrib. Plasma Phys. 39, 97 (1999).

[8] J.M. Caillol, J. Chem. Phys. 111, 6538 (1999). 